\title{
Does Trimethoprim-Sulfamethoxazole prophylaxis induce myelosuppression in primary immune deficiency disease patients; A retrospective, 3 groups comparative study
}

\author{
Reem Elajez, ${ }^{1}$ Sabha Nisar, ${ }^{1}$ Mehdi Adeli1,2,3
}

\begin{abstract}
Background: The possible myelosuppression side effect of Trimethoprim-Sulfamethoxazole (TMP-SMX) on primary immune deficiency (PID) patients has not been established yet.
\end{abstract}

Objective: Identify if the PID patients are at higher risk of developing myelosuppression secondary to the use of TMPSMX.

Method: Retrospective, three groups study, of PID patients (on and off TMP-SMX prophylaxis) and urinary tract infection (UTI) patients received prophylaxis TMP-SMX. Data about CBC results (WBC, ANC, Lymphocytes, RBC, Hemoglobin, and Platelet counts) at baseline, first, and maximum myelosuppression observed during the period of TMP-SMX administration were collected.

Results: A total of 122 patients were included in this study (41 PID patients on TMP-SMX prophylaxis, 45 PID patients not on TMP-SMX prophylaxis, and 36 UTI patients on prophylaxis TMP-SMX). There are significant differences noticed in the percentage of patients who developed clinical myelosuppression (i.e. less than normal value for age) in ANC (39.0\% vs. $8.9 \%$ vs. $16.7 \%, p=0.002)$, RBC ( $36.6 \%$ vs. $13.3 \%$ vs. $13.9 \%, p=0.014)$, WBC $(41.5 \%$ vs. $13.3 \%$ vs. $13.9 \%$, $p=0.003)$, and platelet $(24.4 \%$ vs. $15.6 \%$ vs. $2.8 \%, p=0.028)$ in group 1,2 , and 3 , respectively. Significant difference in myelosuppression between the groups was most likely due to the combination of TMP-SMX effect on PID patients rather than the disease or the drug itself.

Conclusion: Primary immune deficiency (PID) patients are at higher risk of developing myelosuppression secondary to TMP-SMX prophylaxis (especially ANC) comparing to immune-competent patients or other PID patients who did not receive prophylactic TMP-SMX. Future larger prospective study is required to confirm this association.

Key words: TMP-SMX; myelosuppression; primary immune deficiency; prophylaxis, Neutropenia

From:

${ }^{1}$ Hamad Medical Corporation

2 Sidra Medical and Research Center

3 Weill Cornell Medicine in Qatar

\section{Introduction}

Trimethoprim-sulfamethoxazole (TMP-SMX), also known as co-trimoxazole, is a combination of two antimicrobial agents that act synergistically to treat a variety of bacterial infections. ${ }^{1,2}$ It is considered the drug of choice for the treatment and prevention of Pneumocystis jiroveci (carinii) pneumonia (PCP). ${ }^{3-6}$ TMP-SMX is a well-tolerated drug where adverse drug reactions occur in only $6-8 \%$ of patients. ${ }^{7}$ One of the serious TMP-SMX side effect is myelosuppression,
Corresponding author:

Reem Elajez

E-mail: relajez@hamad.qa

which is believed to be due to its anti-folate DNA suppression mechanism. ${ }^{8-10}$

Few studies highlighted and reported the myelosuppression or hematologic abnormalities associated with TMP-SMX use. One study showed that neutropenia occurred in $34 \%$ and thrombocytopenia in $12 \%$ of children who received oral TMP-SMX. ${ }^{11}$ Another study evaluated 120 children found that neutropenia appeared only in those who were treated with 
TMP-SMX. ${ }^{12}$ Unfortunately, this side effect also occurred at the prophylactic dose used for different indications (e.g., Lymphocytic leukemia, and urinary anomalies patients). ${ }^{13-16}$ For example, $30 \%$ of children with urinary tract anomalies treated with prophylactic TMP-SMX for more than a month had developed neutropenia. ${ }^{17}$

Primary Immunodeficiency Diseases (PIDs) are a group of chronic heterogeneous, mainly childhood, inherited disorders with increased susceptibility to infections. ${ }^{18}$ Infectious diseases play a significant role in determining the outcome of PD patients due to their significant impact on both morbidity and mortality. ${ }^{18-20}$ Thus, the application of prophylactic medications is often necessary, where TMP-SMX is the most common recommended prophylactic antibiotic in PID patients. ${ }^{18,20}$ In the United States, adjunctive prophylaxis with TMP-SMX is used in approximately $50 \%$ of patients with agammaglobulinemia, while $14 \%$ in Europe. ${ }^{21}$ A French cohort of 60 patients showed that 54 (90\%) received TMP-SMX as prophylaxis. ${ }^{22}$ In Qatar, the estimated prevalence of PID was found to be 4.7 per 100,000 children, where most of them took TMP-SMX prophylaxis at one point in their life. ${ }^{23}$

It has been found that much higher frequency (up to $50 \%)$ of the myelosuppressive effect of TMP-SMX reported in patients with acquired immune deficiency syndrome (AIDS); most probably due to their impaired immune system. ${ }^{24-26}$ So, as PID patients have some degree of immune deficiency; the percentage of iatrogenic myelosuppression due to TMPSMX prophylaxis is suspected to be higher than in other immune-competent patients studied in previous trials. ${ }^{11-17}$ Therefore, this study aims to evaluate the safety profile of TMP-SMX prophylaxis in patients with PID with regards to myelosuppression effect, which is the first study done of its kind.

\section{Methods}

The Primary objective of this research was to identify if the primary immune deficiency patients are at higher risk of developing myelosuppression secondary to TMP-SMX prophylaxis. The secondary objectives were: 1) categorize the severity of iatrogenic myelosuppression effect of TMP-SMX prophylaxis, 2) identify the mean duration and extent of suppression (cell count difference) observed in patients who developed myelosuppression, and 3) explore the difference in myelosuppression observed in different PID types.

\section{Study Design and Participants:}

This was a retrospective study comparing three groups of patients: 1) Group 1 (PID+TMP-SMX): Primary immune deficiency (PID) patients who received prophylactic TMPSMX, 2) Group 2 (PID): Primary immune deficiency (PID) patients who did not receive prophylactic TMP-SMX, and 3) Group 3 (UTI+TMP-SMX): Immune-competent patients who received prophylactic TMP-SMX for urinary tract infection (UTI)

This study was conducted at Hamad General Hospital (HGH) in Qatar, which was the main and only tertiary hospital in Qatar following PID patients at that time. The patient list was generated and identified differently according to the group. Hence, for group 1 \& 2; medical records department identified PID patients using PID diagnostic ICD codes. All PID patients diagnosed over a span of 30 years, in the period from 01/01/1985 to 10/12/2015 were initially included. This long period was selected as our target population was small and the estimated cases are inconsequential in number. On the other hand, for group 3; pharmacy electronic system was used to generate list of patients being prescribed TMPSMX for more than a week. The generated patient list was screened to remove any duplicate, PID patients, and patients who received a treatment dose of TMP-SMX. Medical records of potentially included patients were briefly skimmed to confirm the indication of TMP-SMX. Only patients who received TMP-SMX as UTI prophylaxis (i.e. patients with recurrent UTI, vesicoureteral reflux (VUR) grade 4 and above) were included.

Identified patients in all groups were screened for eligibility based on the pre-set inclusion \& exclusion criteria as following: inclusion criteria: 1) received TMP-SMX at prophylactic dose $(5 \mathrm{mg} / \mathrm{kg} /$ day for PID and $2 \mathrm{mg} / \mathrm{kg} /$ day for UTI, where dose based on trimethoprim component) for at least 1 week, and 2) available $\mathrm{CBC}$ results at baseline and after the start point. The start point for groups $1 \& 3$ was "the starting date of TMP-SMX prophylaxis"; while for group 2 was "the date of PID diagnosis". On the other hand; exclusion criteria were: 1) concurrent use of other medication that could affect the result (mainly chemotherapy and colony-stimulating factor), 2) underwent Bone Marrow Transplantation (BMT), 3) baseline myelosuppression and 4) unavailability of $\mathrm{CBC}$ result either at baseline or thereafter.

\section{Data collection and measures:}

Data were extracted manually from the patient's medical records (both paper and computer-based records) using a standardized data collection form. The process of data reviewing involved physicians' and nurses' notes, medication prescriptions, medication administration charts, lab results, and any other documents that helped in completing the data collection sheet (e.g. abroad medical reports). The data collection form comprised of 3 sections; 1) Demographic section: patient's age, gender, type of PID, other concurrent medications/conditions, 2) TMP-SMX dosage regimen: start date, dose, and duration, and 3) $\mathrm{CBC}$ results (White Blood Cell (WBC), Absolute Neutrophils Count (ANC), Lymphocyte, Red Blood Cell (RBC), Hemoglobin, and Platelet counts) at baseline, at first, and at maximum myelosuppression. First and maximum myelosuppressions were defined as: first dropped and maximally dropped values, respectively. CBC readings were chosen to be within the duration of TMP-SMX use or within 1 year, whichever is shorter.

Normal CBC values for the patient's age were set using the Harriet lane handbook. ${ }^{27}$ Only suppression seen below the normal limit per the patient's age is considered and labeled as "clinical myelosuppression". The severity grade of these myelosuppressions was then determined based on National Cancer Institute Common Terminology Criteria for adverse events. $^{27}$ 


\section{Ethical consideration}

This study got approval from Hamad Medical Corporation -Medical Research Center (HMC-MRC) in Qatar. Quality of data (review of completeness, data verification and accuracy, security, and confidentiality of data) had been performed and maintained by lead research investigator. All patients' information kept confidential in a password-protected computer, which could only be accessed by the study research investigators.

\section{Statistical Analysis}

Qualitative and quantitative data values were expressed as frequencies along with percentages and mean $\pm \mathrm{SD}$. Descriptive statistics were used to summarize demographic and all other characteristics of the participants. Associations between two or more qualitative or categorical variables were assessed using chi-square test. For small cell frequencies, the chi-square test with continuity correction factor or Fisher's exact test was applied. Pictorial presentations of the key results were made using appropriate statistical graphs. Relationship between two quantitative variables of clinical suppression incidence and different patient factors (i.e. age, PID type, gender) was examined using Pearson's correlation coefficients. Besides, other appropriate univariate and multivariate logistic regression analyses were used to assess and quantify the effect of different factors and covariates on clinical suppression in different cell lines. A two-sided $P$ value $<0.05$ was considered to be statistically significant. All statistical analyses were done using statistical package SPSS, version 22.0 (IBM Corporation, Armonk, NY).

\section{Results \\ Participants}

The study was started with reviewing 280 potential included patients shortlisted from meticulous search in the medical records and pharmacy medication list. A final list of 122 patients was formed while the remaining 158 patients were excluded for different reasons (e.g. unavailability of data before or during the medication period, concurrent administration of chemotherapy, immunodeficiency diagnosis was still under investigation or history of bone marrow transplantation). Out of the final 122 included patients, group 1 had 41 patients, group 2 had 45 patients, and group 3 had 36 patients (Table 1). Among the three groups, the mean patients' age ranged from birth to 26.5 years. Comparable percentages of males and females were found in groups $1 \& 3$, whereas males $(73.3 \%)$ outnumbered the females $(26.7 \%)$ in group 2. Although there is an observed difference in gender between groups, gender was tested and found not to have any statistically significant effect on clinical suppression for all cell lines.

As included PID patients had different PID diagnosis; it was classified into six main types; 1) Immune dysregulation, 2) Congenital Malformation associated with immune dysfunction, 3) Humoral Immunodeficiency, 4) Innate Immune defect neutrophil function/Migration disorder, 5) T cell defect, and 6) Ataxic Telangiectasia. Types of PID existed in group 1 patients were different than group 2, where "innate immune defect" had the most frequent type $(43.9 \%)$ in group 1, while "humoral immunodeficiency" was in group 2 (35.6\%).

Table 1. Demographics

\begin{tabular}{|c|c|c|c|}
\hline Group & $\begin{array}{c}\text { Group } 1 \\
\begin{array}{c}(\text { PID + TMP-SMX) } \\
(\mathrm{n}=41)\end{array}\end{array}$ & $\begin{array}{l}\text { Group } 2 \\
\text { (PID) } \\
(n=45)\end{array}$ & $\begin{array}{c}\text { Group } 3 \\
(\mathrm{UTI}+\mathrm{TMP}-\mathrm{SMX}) \\
(\mathrm{n}=36)\end{array}$ \\
\hline Age in years (mean $\pm S D$ ) & $4.3 \pm 4.1$ & $4.4 \pm 5.9$ & $3.4 \pm 3.9$ \\
\hline $\begin{array}{l}\text { Duration of TMP-SMX in years } \\
\text { Mean (range) }\end{array}$ & $\begin{array}{c}2.3 \\
(0.1-7)\end{array}$ & N/A & $\begin{array}{c}2.4 \\
(0.25-10)\end{array}$ \\
\hline \multicolumn{4}{|l|}{ Gender, n (\%) } \\
\hline Male & $23(56.1)$ & $33(73.3)$ & $16(44.4)$ \\
\hline Female & $18(43.9)$ & $12(26.7)$ & $20(55.6)$ \\
\hline \multicolumn{4}{|l|}{ Type of PID, n (\%) } \\
\hline Innate immune defect neutrophil function & $18(43.9)$ & $8(17.8)$ & $\mathrm{N} / \mathrm{A}$ \\
\hline T cell defect & $12(29.3)$ & $10(22.2)$ & $\mathrm{N} / \mathrm{A}$ \\
\hline Humoral Immunodeficiency & $4(9.8)$ & $16(35.6)$ & N/A \\
\hline Ataxic Telangiectasia & $3(7.3)$ & $10(22.2)$ & $\mathrm{N} / \mathrm{A}$ \\
\hline Immune Dysregulation & $2(4.9)$ & $1(2.2)$ & N/A \\
\hline Congenital Malformation & $2(4.9)$ & $0(0)$ & N/A \\
\hline
\end{tabular}




\section{Primary outcome}

A general trend of suppression was seen in all cell lines of the three groups, specifically in WBC, lymphocytes, and platelets. In group 1, ANC was suppressed in $80.5 \%$ of the patients, which is the highest compared to the other two groups $(62.2 \%, 61.1 \%$, respectively). However, only hemoglobin showed to have a statistically significant difference between the three groups with the highest percentage seen in group $2(p=0.003)$ (Figure 1 ).

Clinical myelosuppression (i.e. suppression in cell line below the normal limit for the patient's age) was more prominent in group 1, where it had the maximum clinical suppression in the entire cell lines. However, suppression in four out of the six cell lines found to have statistically significant differences between the three groups. These cell lines include
ANC (39.0\% vs. $8.9 \%$ vs. $16.7 \%, p=0.002)$, RBC (36.6\% vs. $13.3 \%$ vs. $13.9 \%, p=0.014)$, WBC $(41.5 \%$ vs. $13.3 \%$ vs. $13.9 \%$, $p=0.003)$, and platelet $(24.4 \%$ vs. $15.6 \%$ vs. $2.8 \%, p=0.028)$ in group 1, 2, and 3, respectively (Figure 2). Patients in group 1 were at higher risk of having clinical suppression of WBC by 5.7 folds compared to group 3 (confidence interval [CI], 1.77518.547), and ANC by 2.5 folds compared to group 3 (confidence interval [CI], 0.787-8.009). Odds of RBC clinical suppression were 3 times higher among group 1 compared to group 3 (95\% confidence interval [CI] 0.733-12.273).

As Innate immune defect neutrophil function disorders (IIDNFD) are not associated with myelosuppression as part of the disease itself, we believed that suppression seen in this group were most likely due to TMP-SMX effect rather than the disease itself. Thus, when comparing myelosuppression

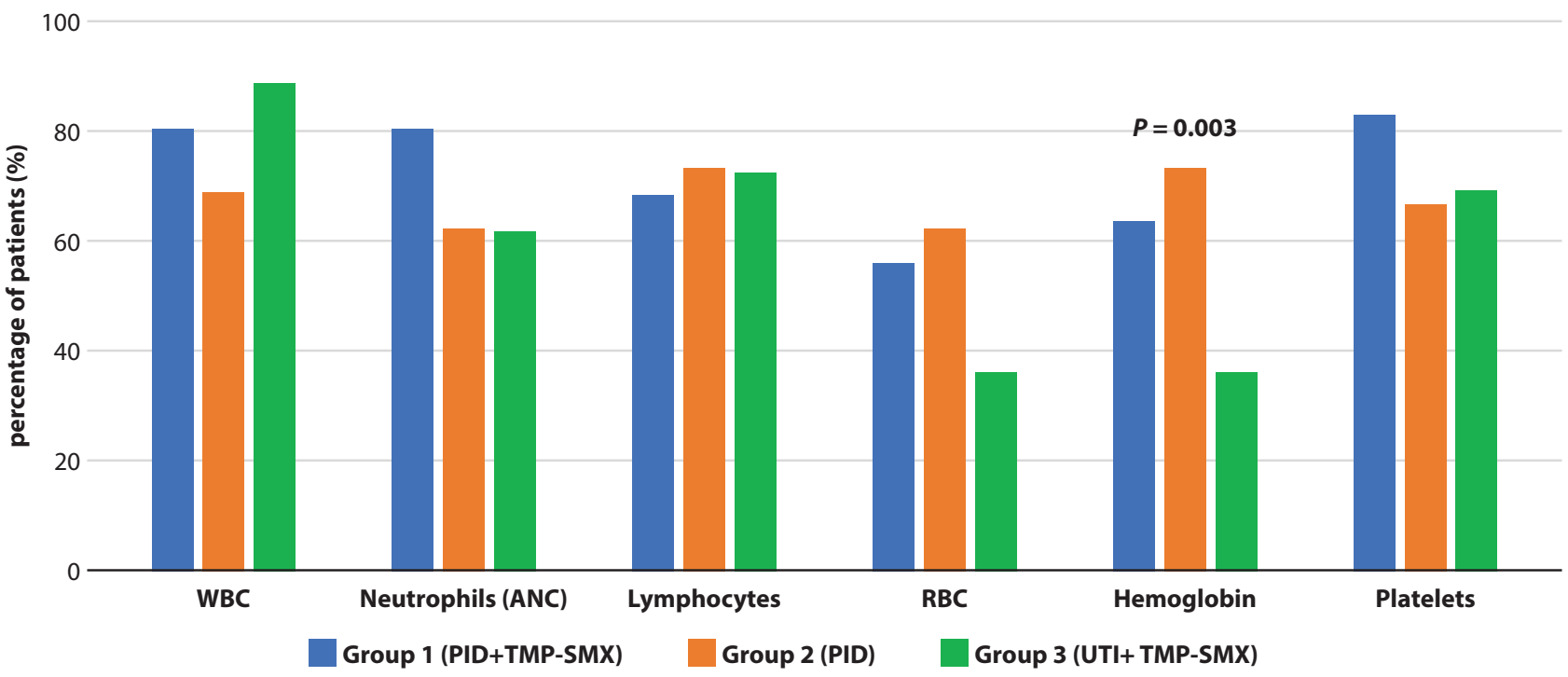

Figure 1. Percentage of patients who developed suppression across different cell lines in the three comparative groups. Only hemoglobin found to be significant, with $P$-value < 0.05. WBC: White Blood Cells, ANC: Absolute Neutrophils Count, RBC: Red Blood Cells, PID: Primary Immune Deficiency, TMP-SMX: Trimethoprim-Sulfamethoxazole, UTI: Urinary tract infection

$$
100
$$

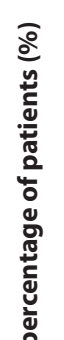$$
80
$$$$
60
$$$$
\text { ठั }
$$$$
40
$$$$
P=0.002
$$$$
P=0.014
$$$$
20
$$

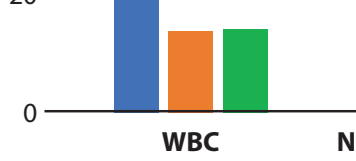

Neutrophils (ANC)

Lymphocytes

Group 2 (PID)

$P=0.014$

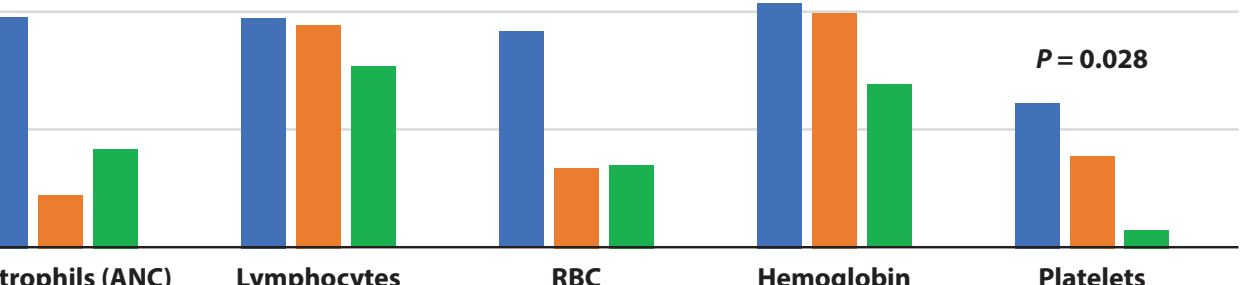

Group 1 (PID+TMP-SMX)

Group 3 (UTI+ TMP-SMX)

Figure 2. Percentage of patients who developed clinical myelosuppression across different cell lines in the three comparative groups. Only significant $p$-value $(<0.05)$ presented in the graph. WBC: White Blood Cells, ANC: Absolute Neutrophils Count, RBC: Red Blood Cells, PID: Primary Immune Deficiency, TMP-SMX: Trimethoprim-Sulfamethoxazole, UTI: Urinary tract infection 
in IIDNFD patients who received TMP-SMX from group 1 $(\mathrm{n}=18)$ to group 3 (Control group), it showed steeper suppression in WBC $(22.2 \%$ vs $13.9 \%)$, RBC count $(22.2 \%$ vs $13.9 \%)$, and platelet count (16.7\% vs $2.8 \%)$. Myelosuppression in hemoglobin was found to be the same for both groups $(27.8 \%)$. However, none of the cell lines showed statistically significant differences between the two groups.

\section{Secondary Outcomes}

The severity of myelosuppression was graded on a scale from 0 to 4 according to the criteria set by National Care Institute Common Terminology Criteria for Adverse Events. ${ }^{28}$ Grade 4 is the maximum severity of suppression while grade 1 is the lowest suppression. In addition to a higher number of patients developed neutropenia (ANC clinical suppression) in group $1(n=16)$ compared to other groups, neutropenia seen in group 1 patients was more severe than the other patients in groups $2 \& 3$ (Table 2 ). The severity of grade 4 (i.e. cell count $<500 / \mu \mathrm{l}$ ) was seen in 7 patients from group 1 whereas only 1 patient from each other groups had grade 4 severity.
No patient in any group developed neutropenia of grade 1 severity. Thrombocytopenia of grade 1 severity was found in 5 patients in each group 1 and 2, whereas grade 4 severity was seen in only one patient of group 2 .

For group 1, suppression among different cell lines was first noted after an average of 2.4 months, while the maximum suppression occurred after an average of 3.7 months. Lymphocyte was the last cell line to be suppressed as it took the longest time to show first/maximum suppression (2.8/5 months), whereas ANC was the fastest line to be suppressed (i.e. first/maximum suppression occurred at an average of 1.7/2.8 months). In contrast to group 1, group 2 and 3 demonstrated a longer mean duration for first myelosuppression (3.5 and 4.5 months) and a maximum suppression with an average of 5 and 5.5 months for group 2 and 3, respectively.

Mean count difference from baseline till the maximum suppression was found to be the same in the three groups for WBC $\left(4.6 \times 10^{3} / \mathrm{mL}\right)$, and hemoglobin $(2.1 \mathrm{~g} / \mathrm{dl})$. It had been noticed that the mean difference in cell count between

Table 2. Severity grade of neutropenia ${ }^{1}$

\begin{tabular}{|c|c|c|c|c|c|}
\hline Group & $\begin{array}{c}\text { Patients who developed } \\
\text { neutropenia }\end{array}$ & $\begin{array}{c}\text { Grade } 1 \\
<\mathrm{LLN}^{2} \text { to } 1500 / \mu \mathrm{l}\end{array}$ & $\begin{array}{c}\text { Grade } 2 \\
1000 \text { to } 1500 / \mu l\end{array}$ & $\begin{array}{c}\text { Grade } 3 \\
500 \text { to } 1000 / \mu l\end{array}$ & $\begin{array}{l}\text { Grade } 4 \\
<500 / \mu l\end{array}$ \\
\hline $\begin{array}{l}\text { Group } 1(\mathrm{n}=41) \\
\text { UTI + TMP-SMX }\end{array}$ & 16 & 0 & 5 & 4 & 7 \\
\hline $\begin{array}{l}\text { Group } 2(\mathrm{n}=45) \\
\text { PID }\end{array}$ & 4 & 0 & 1 & 2 & 1 \\
\hline $\begin{array}{l}\text { Group } 3(n=36) \\
\text { UTI + TMP-SMX }\end{array}$ & 6 & 0 & 4 & 1 & 1 \\
\hline
\end{tabular}

${ }^{1}$ Classified according to National Cancer Institute Common Terminology Criteria for adverse events

${ }^{2}$ LLN: Lower Limit of Normal

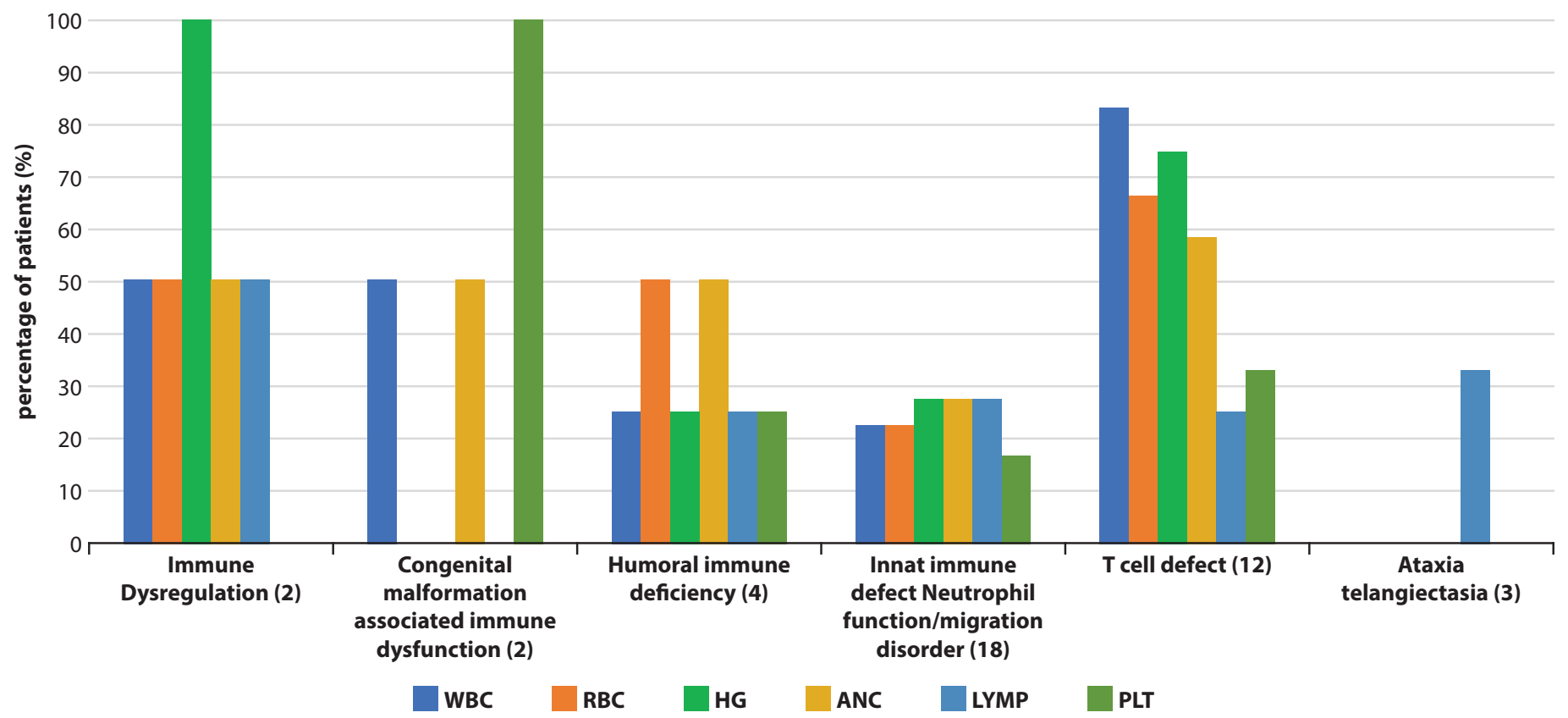

Figure 3. Percentage of patients who developed myelosuppression of cell lines in different Primary Immune Deficiency (PIDs) types from Group 1 (PID on TMP-SMX prophylaxis). WBC: White Blood Cells, RBC: Red Blood Cells, HG: Hemoglobin, ANC: Absolute Neutrophils Count, LYMP: lymphocytes, PLT: Platelets 
baseline and maximum suppression was smallest in group 2 for platelets $\left(85 \times 10^{3} / \mathrm{mL}\right.$ vs. $150 \times 10^{3} / \mathrm{mL}$ for other two groups) and was biggest in group 3 for lymphocytes $\left(2.9 \times 10^{3}\right.$ cells $/ \mu \mathrm{L}$ vs. $1.7 \times 10^{3}$ cells $/ \mu \mathrm{L}$ for other two groups). However, as an overall, there were no significant variances between the three groups in terms of the mean count differences from baseline to first or baseline to maximum suppression for all cell lines.

In group 1, the maximum type of PID patients who developed ANC and WBC suppression was patients with "T cell defect" (58.3\% and $83.3 \%$, respectively). Hemoglobin was reduced in $100 \%$ of patients with "immune dysregulation", whereas none of the patients with "congenital malformation associated immune dysfunction" or "Ataxic telangiectasia" had anemia. Interestingly, patients with "Ataxic telangiectasia" only expressed suppression in one cell line, which was lymphocyte (33\%). Patients with "Innate immune defect neutrophil function/migration disorder" found to have myelosuppression in all cell lines with an average (Figure 3).

\section{Discussion}

Up to our best knowledge, this study is the first study evaluating the myelosuppression effect of TMP-SMX prophylaxis in PID patients. In this retrospective study, myelosuppression effect of TMP-SMX prophylaxis was investigated among three groups for six different cell lines. The primary finding of this study showed that myelosuppression was predominantly instituted amongst the PID patients on TMPSMX prophylaxis. Likewise, the severity of suppression was found to be highest in PID patients receiving TMP-SMX compared to other patients.

TMP-SMX induced myelosuppression is superimposed with an existing immunodeficiency. Several mechanisms have been asserted by which the medication produces these side effects. TMP-SMX can cause myelosuppression either as part of drug hypersensitivity syndrome or folate deficiency. ${ }^{29-30}$ Since PID patients have a degree of myelosuppression (mainly due to defects in immune system development and/or function), this makes them at higher risk of aggravated myelosuppression when taking TMP-SMX. This added risk led to the higher suppression seen in cell lines of group 1 patients compared to the other two groups. We validated this hypothesis by comparing group 1 with the other two control groups (i.e. groups 2 and 3), where factors like PID disease and TMP-SMX administration were controlled in each group. Furthermore, we also looked to PID types which are not associated with any myelosuppression as part of the disease pathogenesis (like "innate immune defect - neutrophil function disorders"). ${ }^{31}$ Since these patients had also experienced myelosuppression while they were on TMP-SMX prophylaxis; the suppression hee is highly suspected to be purely from the drug's effect.

Our study demonstrates that $\mathrm{WBC}, \mathrm{ANC}, \mathrm{RBC}$, and platelets were statistically significant depressed in group 1 compared to other groups. Previous studies were mainly focusing on ANC rather than other cell lines. Three studies had described neutropenia that associated with TMP-SMX therapy in the immune-competent population received TMP-SMX for UTI indication. ${ }^{11-12,17}$ A previous study evaluated children treated with oral TMP-SMX found that neutropenia (ANC $\leq 1,500 / \mu \mathrm{l})$ was developed in 17 out of $50(34 \%) .{ }^{11}$ Comparably, Principi $\mathrm{N}$ et al. deduced the incidence of neutropenia to be $35 \%$ in 120 children treated with TMP-SMX. ${ }^{12}$ These results were reported using the treatment dose of TMP-SMX, which is much higher than the prophylaxes dose. Only one study evaluated the effect of prophylactic TMP-SMX in children affected by urinary tract anomalies $(n=27)$ and found that $27 \%$ developed neutropenia. ${ }^{17}$ Nevertheless, our study found a lower incidence of neutropenia in patients who were at UTI prophylaxis group (16.7\%). For neutropenia incidence in immunodeficiency patients, there was no previous study in PID patients to compare with. However, AIDS patients are immunodeficiency patients, where the effect of prophylactic TMP-SMX was previously studied. Toure $S$ et al. describes the incidence of neutropenia in a 6-year cohort of AIDS adults receiving TMP-SMX prophylaxis. ${ }^{32}$ Out of 533 AIDS patients with normal baseline ANC, 58.7\% developed neutropenia of ANC $<1500 / \mu \mathrm{l}$. This result was quite higher than what was found in our study, where $39 \%$ of the PID children receiving prophylactic TMP-SMX developed neutropenia. This difference could be due to the disease pathogenesis itself, or other concurrent medications that usually AIDS patients were taking.

For platelet suppression, thrombocytopenia was only reported to be developed in six (12\%) out of 50 children received TMP-SMX as UTI treatment. ${ }^{11}$ This was higher than the result of our study, where platelet suppression was $2.8 \%$ in UTI group. An explanation of this difference could be due to the difference in the TMP-SMX dose between treatment and prophylaxis.

The severity of observed neutropenia was assessed according to the National Cancer Institute of Common Terminology Criteria for Adverse Events. ${ }^{28}$ Advanced severity levels were found more frequently in group 1 (PID patients on TMPSMX) compared to the other two groups. Although the TMPSMX doses used in groups 1 was higher than group 3 (5 vs. 2 $\mathrm{mg} / \mathrm{kg} /$ day), the previous studies showed that myelosuppression is not a dose-dependent side effect, and it could occur regardless of the used dose. ${ }^{11-12,17}$ As an accurate representation of severity level, we tried including data for clinical manifestation in cases of severe suppression, but unfortunately, it was difficult to capture such information from the physician's notes respectively.

Interestingly, neutrophil was the fastest line to be suppressed amongst all cell lines; likewise, it took the least duration to reach maximum suppression. This could be explained by the short lifespan of neutrophils. In contrast, lymphocytes took the longest time. Comparable to our study, a previous study showed that late suppression was evident in lymphocytes whereas ANC took the shortest time to get suppressed. ${ }^{12}$

This study met with a few limitations. First, as it was exclusively a retrospective study, the information heavily relied on pre-existing data records, which were a detriment in cases of missing data. Attributable to it is retrospective nature; many essential data could not be assessed accordingly. For example, the patient's compliance with TMP-SMX could not be assured; thus, we merely depended on the frequency of dispensing medication as an indicator of patient compliance. 
Furthermore, the physician's actions toward myelosuppression development (e.g. discontinuation of therapy or switching to other alternatives) could not always be assessed. The second limitation was some confounding factors couldn't be totally controlled (i.e. concomitant infections, nutritional deficiencies, and other concurrent diseases/medications). However, patients received medications that can significantly alter the results (e.g. Granulocyte-colony stimulating factor (GCSF) and chemotherapy) were excluded. Third limitation was infrequent blood investigations for the control group (group 3) as compared to the other two groups, which made comparable assessments harder.

On the contrary, the study has substantial strengths that are worth mentioning. The study design constituted of three groups for comparison, which backed in determining the myelosuppression effect of TMP-SMX in PID patients. To ensure that the effect seen in group 1 was due to the medication rather than the disease itself, a subgroup analysis was performed and group 2 was included. Also, none of the groups were on folic acid. Moreover, clinical myelosuppression (suppression below the normal limit per the patient's age) was assessed rather than just looking for suppression. Additionally, since HGH was the only tertiary hospital following PID patients in Qatar, our targeted population included all PID patients in Qatar. Last but not least, this study is the first study to assess the effect of TMP-SMX on PID patients.

There is a future need to further elaborate this retrospective study into a large prospective study with a complete follow-up of all groups. The confounding factors (such as infections, nutritional deficiencies, general health, other concurrent medications, and drug interaction with TMP-SMX) need to be taken into account. Myelosuppression effect of TMP-SMX must also be studied on different types of PIDs which were not found in this region.

\section{Conclusion}

In conclusion, the myelosuppressive effect of TMP-SMX is amplified in primary immunodeficiency patients (particularly ANC and platelet) resulting in possible serious complications with long term prophylaxis. The potential myelosuppressive effect varies among different types of PIDs. When prescribing TMP-SMX to PID patients, risks and benefits must be weighed accordingly. Physicians must closely monitor the complete blood count at regular intervals and dose/ duration must be adjusted accordingly.

\section{Acknowledgement}

We would like to acknowledge Dr. Kareem Basharat for his significant contribution in data collection.

\section{Funding source}

This study was funded by Hamad Medical Corporation -Medical Research Center (HMC-MRC) in Qatar

\section{Conflicts of interest}

The authors of this study have nothing to disclose concerning the possible financial or personal relationship with commercial entities that may have a direct or indirect interest in the subject matter of this study

\section{References}

1. Kalkut G. Sulfonamides and trimethoprim. Cancer Invest. 1998;16(8): 612-5.

2. World Health Organization [Internet]. Geneva: WHO; c2014 [cited 2014 Apr 22]. WHO Model List of Essential Medicines; [about 2 screens]. Available from: https://www.who.int/medicines/publications/essential medicines/en/.

3. Gleckman R, Blagg N, Joubert DW. Trimethoprim: mechanisms of action, antimicrobial activity, bacterial resistance, pharmacokinetics, adverse reactions, and therapeutic indications. Pharmacotherapy. 1981;1(1):14-20.

4. Hong YL, Hossler PA, Calhoun DH, Meshnick SR. Inhibition of recombinant Pneumocystis carinii dihydropteroate synthetase by sulfa drugs. Antimicrob Agents Chemother. 1995;39(8):1756-63.

5. Howe RA, Spencer RC. Cotrimoxazole. Rationale for re-examining its indications for use. Drug Saf. 1996;14(4):213-8.

6. Gleckman R, Gantz NM, Joubert DW. Intravenous sulfamethoxazole -trimethoprim: pharmacokinetics, therapeutic indications, and adverse reactions. Pharmacotherapy. 1981;1(3):206-11.

7. Jick H, Derby LE. A large population-based follow-up study of trimethoprim-sulfamethoxazole, trimethoprim, and cephalexin for uncommon serious drug toxicity. Pharmacotherapy. 1995;15(4):428-32.

8. Kocak Z, Hatipoglu CA, Ertem G, Kinikli S, Tufan A, Irmak H, et al. Trimethoprim-sulfamethoxazole induced rash and fatal hematologic disorders. J Infect. 2006;52(2):e49-52.

9. Hellmann A, Nering I. Complete bone marrow suppression and megaloblastic anemia as a result of long-term treatment with co -trimoxazole. Pol Tyg Lek. 1977;32(46):1801-2.

10. Parajuli P, Ibrahim AM, Siddiqui HH, Lara Garcia OE, Regmi MR. Trimethoprim-sulfamethoxazole Induced Pancytopenia: A Common Occurrence but A Rare Diagnosis. Cureus. 2019;11(7):e5071.

11. Asmar BI, Maqbool S, Dajani AS. Hematologic abnormalities after oral trimethoprim-sulfamethoxazole therapy in children. Am J Dis Child. 1981;135(12):1100-3.

12. Principi N, Marchisio P, Biasini A, Dalla Villa A, Biasini G. Early and late neutropenia in children treated with co-trimoxazole (trimethoprim -sulfamethoxazole). Acta Paediatr Scand. 1984;73(6):763-7.

13. Schey SA, Kay HE. Myelosuppression complicating co-trimoxazole prophylaxis after bone marrow transplantation. Br J Haematol. 1984; 56(1):179-80.

14. Bow EJ, Louie TJ, Riben PD, McNaughton RD, Harding GK, Ronald AR. Randomized controlled trial comparing trimethoprim/ sulfamethoxazole and trimethoprim for infection prophylaxis in hospitalized granulocytopenic patients. Am J Med. 1984;76(2):223-33.

15. Dworkin MS, Williamson J, Jones JL, Jonathan E.Prophylaxis with trimethoprim-sulfamethoxazole for human immunodeficiency virus -infected patients: impact on risk for infectious diseases. Clin Infect Dis. 2001;33(3):393-8.

16. Woods WG, Daigle AE, Hutchinson RJ, Robison LL. Myelosuppression associated with co-trimoxazole as a prophylactic antibiotic in the maintenance phase of childhood acute lymphocytic leukemia. J Pediatr. 1984;105(4):639-44.

17. De Manzini A, Peratoner L, Lepore L. Neutropenia caused by low-dose trimethoprim-sulfamethoxazole in children with chronic pathology of the urinary tract. Pediatr Med Chir. 1990;12(1):49-51.

18. Kuruvilla M, de la Morena MT. Antibiotic prophylaxis in primary immune deficiency disorders. J Allergy Clin Immunol Pract. 2013;1(6):573-82.

19. Papadopoulou-Alataki E, Hassan A, Davies EG. Prevention of infection in children and adolescents with primary immunodeficiency disorders. Asian Pac J Allergy Immunol. 2012;30(4):249-58.

20. Aguilar C, Malphettes M, Donadieu J, Chandesris O, Coignard-Biehler $\mathrm{H}$, Catherinot E, et al. Prevention of infections during primary immunodeficiency. Clin Infect Dis. 2014;59(10):1462-70. 
21. Hernandez-Trujillo HS, Chapel H, Lo Re, Notarangelo LD, Gathmann B, Grimbacher B, et al. Comparison of American and European practices in the management of patients with primary immunodeficiencies. Clin Exp Immunol. 2012;169:57-69.

22. Chandesris MO, Melki I, Natividad A, Puel A, Fieschi C, Yun L, et al. Autosomal dominant STAT3 deficiency and hyper-IgE syndrome: molecular, cellular, and clinical features from a French national survey. Medicine (Baltimore). 2012;91:e1-19.

23. Ehlayel MS, Bener A, Laban MA. Primary immunodeficiency diseases in children: 15 year experience in a tertiary care medical center in Qatar. J Clin Immunol. 2013;33(2):317-24.

24. Medina I, Mills J, Leoung G, Hopewell PC, Lee B, Modin G, et al. Oral therapy for Pneumocystis carinii pneumonia in the acquired immunodeficiency syndrome. A controlled trial of trimethoprim -sulfamethoxazole versus trimethoprim-dapsone. N Engl J Med. 1990; 323(12):776-82

25. Van AJ, Vree TB, Koopmans PP, van der Meer JW. Adverse reactions to co-trimoxazole in HIV infection: a reappraisal of the glutathione -hydroxylamine hypothesis. J Antimicrob Chemother. 1996; 37 Suppl B: $55-60$.
26. Gordin FM, Simon GL, Wofsy CB, Mills J. Adverse reactions to trimethoprim-sulfamethoxazole in patients with the acquired immunodeficiency syndrome. Ann Intern Med. 1984;100(4):495-9.

27. Johns Hopkins Hospital (US). The Harriet Lane handbook: A manual for pediatric house officers.20th ed. Philadelphia: Elsevier; 2014

28. National Institutes of Health [Internet]. Bethesda: NIH; c2015 [cited 2015 Oct 22]. Common Terminology Criteria for Adverse Events (CTCAE); [about 2 screens]. Available from: http://evs.nci.nih.gov/ftp1/CTCAE/ CTCAE_4.03_2010-06-468 14_QuickReference_5x7.pdf

29. Rezaei N, Moazzami K, Aghamohammadi A, Klein C. Neutropenia and primary immunodeficiency diseases. Int Rev Immunol. 2009;28(5):335-66.

30. Ho JWH, Juurlink DN. Considerations when prescribing trimethoprim -sulfamethoxazole. CMAJ. 2011;183(16):1851-8.

31. Sergio D. Rosenzweig, Steven M. Holland. Recent Insights into the Pathobiology of Innate Immune Deficiencies. Curr Allergy Asthma Rep. 2011;11(5):369-77.

32. Toure S, Gabillard D, Inwoley A, Seyler C, Gourvellec G, Anglaret X. Incidence of neutropenia in HIV-infected African adults receiving co-trimoxazole prophylaxis: a 6-year cohort study in Abidjan, Côte d'Ivoire. Trans R Soc Trop Med Hyg. 2006;100(8):785-90. 